\title{
POSICIONAMENTO ESTRATÉGICO DE PRODUTOS COM INOVAÇÃO TECNOLÓGICA: O CASO DO PRODUTO WI-FI
}

\section{Márcio de la Cruz Lui}

Mestre em Administração pelo Centro Universitário da FEI

Professor da Universidade Nove de Julho - UNINOVE

marciodlclui@gmail.com (Brasil)

\section{Braulio Oliveira}

Doutor em Administração pela Faculdade de Economia, Administração e Contabilidade da Universidade de São Paulo - FEA/USP

Professor Pesquisador do Centro Universitário da FEI

braulio@fei.edu.br (Brasil)

\section{RESUMO}

Possíveis diferenças existentes no marketing aplicado a produtos com inovação tecnológica requerem estudos específicos acerca do tema. Mas por enquanto poucas pesquisas foram desenvolvidas com esse foco. Assim, este trabalho buscou conhecer o processo de posicionamento de um produto com inovação tecnológica. Além da revisão da literatura pertinente, foi realizado um estudo de caso. Os resultados apontam pequenas diferenças em relação ao modelo tradicional de posicionamento, em especial a necessidade de se definir um posicionamento menos perecível, uma vez que produtos tecnológicos, por sua própria natureza, requerem constantes atualizações.

Palavras-chave: Posicionamento; Inovação tecnológica; Novos produtos. 


\section{INTRODUÇÃO}

Os desafios competitivos exigem que as empresas inovem constantemente, o que pode se dar por meio de novos produtos e serviços (LEVITT, 1960; AAKER, 2001; PORTER, 2004; DAY, 2001; PRAHALAD; HAMEL, 2005). Por seu turno, deve-se considerar que um produto só se torna inovador se for absorvido pelo mercado, do contrário, não passará de mera invenção (STONEMA; DIEDEREN, 1994, p.918; DOGSON; GANN; SALTER, 2008).

O processo de inovação pode ser concluído com maior sucesso se as ferramentas de marketing forem usadas de maneira adequada para que as expectativas sejam atingidas no lançamento de um novo produto ou serviço (ORGANISATION FOR ECONOMIC CO-OPERATION AND DEVELOPMENT - OECD, 2005, p.17). Tanto que "O marketing para produtos inovadores deve ser considerado um ramo da ciência do marketing, graças aos fatores críticos de sucesso singulares quando há tecnologia envolvida". (PARASURAMAN; COLBY, 2002, p.17).

Revisitar as questões sobre posicionamento em mercados dinâmicos - sobretudo em mercados de tecnologia, como é o setor de telecomunicações - pode ser determinante para se encontrar espaços nos quais a concorrência seja menos intensa (PORTER, 2004; SCHUMPETER, 2003) ou, no melhor caso, espaços onde não haja concorrência (KIM; MAUBORGNE, 2005), e essas lacunas possam ser exploradas através de produtos inovadores, ou ainda subsidiar decisões de manter-se na posição atual, reposicionar-se ou até mesmo reposicionar a concorrência, a fim de trazer vantagem competitiva (RIES; TROUT, 2006).

Além disso, durante o seu ciclo de vida, é necessário que se tomem decisões estratégicas assertivas, no sentido de buscar, decodificar e indicar a necessidade de outras inovações, ou novos produtos e serviços que o mercado possa entender como acréscimo de valor (LEVITT, 1990). A palavra inovação é frequentemente usada em conjunto com tecnologia, mas em termos de limitações de conceitos torna-se importante classificar que apesar de haver uma intersecção entre as definições sobre inovação e produtos com inovação tecnológica, o conceito de inovação é muito mais amplo. Uma inovação não precisa ser necessariamente tecnológica ela pode ser, por exemplo, social. Por outro lado a inovação tecnológica pode ser compreendida como produtos ou processos tecnologicamente novos ou melhorias significativas que tenham sido implementadas nos produtos existentes (DRUCKER, 2003 p.41; ARRUDA; VERMULM; HOLLANDA;; 2006, p.13; OECD, 2005, p.35; TORNATZKY; FLEISCHER, 1990; PARASURAMAN; COLBY, 2002) 
Tecnologia é um artefato de aplicação prática ou um conhecimento que permite determinado desenvolvimento ou uso, incluindo conhecimento e capacidade necessária para entrega de funcionalidade e reprodução. A palavra techno significa avanço de conhecimento; a tecnologia pode ser manifestada através de novos produtos, processos ou sistemas. Por outro lado inovação é o sucesso comercial da exploração de novas idéias ela inclui dentre outras coisas tecnologia, conhecimento científico, sistema organizacional, financeiro e atividades de negócios para a introdução comercial de um novo produto ou melhoria de um produto existente, é muito mais abrangente que uma invenção. (PARASURAMAN; COLBY, 2002; DOGSON; GANN; SALTER; 2008; FREEMAN; SOETE, 1997).

Parassuraman e Colby (2002, pg.17) advogam que o marketing para produtos inovadores apresentam um caráter exclusivo, fatores relacionados à construção de relacionamentos satisfatórios com clientes são diferentes quando há tecnologia envolvida.

Os autores apresentam quatro princípios exclusivos do marketing para produtos inovadores:

- Princípio 1: A adoção de tecnologia é um processo distinto. Alguns fatores que foram pesquisados pelos autores geram algumas crenças dos consumidores em relação à tecnologia. Estas podem ser descritas como: níveis variáveis de otimismo em relação a tecnologia, tendência para inovar, desconforto com tecnologia e insegurança. Essas crenças são menos relevantes para produtos não tecnológicos.

- Princípio 2: As inovações de tecnologia exigem estratégias de marketing diferentes. Os processos de adoção, projeto, preço, comunicação, distribuição e assistência técnica são diferentes para clientes que adotam tecnologia. Um usuário de um computador pode, por exemplo, perder ou sofrer com a sua auto estima caso ele não funcione como ele deseja na frente de outras pessoas. O papel dos primeiros adotantes é fundamental, pois os mesmos têm maior confiança e se sentem mais a vontade com o uso da tecnologia, além do que, esforços devem ser direcionados com o objetivo de tornar o produto fácil de usar, além de dar suporte aos novos usuários.

- Princípio 3: Garantir a satisfação do cliente é um desafio maior para produto ou serviço baseado em tecnologia. Os fatores relacionados ao desconhecido e ao novo exigem abordagens mais complexas para alcançar satisfação dos clientes. Serão necessários treinamento e suporte técnico aos usuários com diversos níveis de receptividade e da ajuda exigida do suporte oferecido.

- Princípio 4: Os mercados de tecnologia são regidos pela lei de massa crítica em que, frequentemente, o resultado é do tipo "o vencedor fica com tudo". A conquista da posição 
dominante por uma empresa de tecnologia raramente é ameaçada por outras empresas, a não ser que haja uma tecnologia substituta. Os primeiros a concorrerem com a nova tecnologia podem obter algum sucesso no início, mas o normal é que uma única empresa conquiste à posição de líder absoluto subjugando as demais posições de nicho.

No entanto, por ser uma aplicação relativamente nova, o marketing para produtos inovadores, sobretudo tecnológicos, necessita ser discutido. Shugan (2004), por exemplo, analisa que as pesquisas de marketing relacionadas a tecnologias emergentes se preocupam em antecipar tendências ao invés de pesquisarem as tecnologias do passado. Assim sendo, este trabalho tem por objetivo conhecer o posicionamento de novos produtos com inovação tecnológica. Para tanto, além da revisão da literatura pertinente, foi realizada uma pesquisa de campo, por meio de um estudo de caso, conforme será apresentado mais adiante.

\section{POSICIONAMENTO DE NOVOS PRODUTOS}

O conceito de posicionamento é bastante discutido no marketing e há uma concordância a respeito da sua importância tanto no meio acadêmico quanto no meio empresarial, porém existem muitos pontos de vista a respeito da definição de posicionamento. Segundo Ogilvy (1985, p.12), “o curioso conceito de posicionamento é muito comum entre os especialistas de marketing, mas não existem dois que concordem sobre o seu significado". Aaker e Shansby (1982, p.56) também analisam que "posicionamento significa diferentes coisas para diferentes pessoas", e complementam: "para alguns são decisões de segmentação, para outros trata-se de uma questão de imagem e ainda para outros ele se refere a quais características do produto será enfatizada”. Já na visão de Bainsfair (1990, p.18) "posicionamento é uma daquelas palavras que todo mundo usa, mas que poucas pessoas entendem". O fato é que posicionamento é uma evolução dos conceitos de marketing em função da evolução dos mercados e da concorrência.

Maggard (1976, p. 64) entende que posicionamento não é só um processo de comunicação, uma vez que envolve outros processos de marketing da empresa. Assim, pode ser considerado em duas perspectivas: a operacional e a estratégica (MÜHLBACHER et al., 1994, p. 288). Além disso, para Kalafatis et al. (2000, p. 14), o posicionamento envolve definição, modificação e monitoramento da percepção dos consumidores em relação a determinado objeto de mercado. 
Há uma relação intensa entre posicionamento e estratégia, o posicionamento relaciona-se com a estratégia do ponto de vista da continuidade do processo de segmentação e pela busca de posições de mercado onde as forças concorrenciais possam ser defendidas ou tenham menor intensidade com possibilidade de buscar posições ainda não ocupadas, ou seja, onde não haja concorrência (TOLEDO; HEMZO, 1991, p. 4; PORTER, 2004, p. 32).

Seu processo inclui tanto a análise do ambiente externo - avaliando as ameaças e oportunidades -, quanto à análise do ambiente interno - avaliando as forças e as fraquezas (AAKER, 2001; PORTER, 2004; GHEMAWAT, 2007).

Segundo Mattar et al. (2009, p. 354) o posicionamento não está apenas relacionado à proposição e à entrega de valor ao cliente de forma diferenciada da concorrência, mas também está baseado na crescente dificuldade em se obter a atenção do consumidor e a oferta de um produto com benefício único.

Em termos de processo, Oliveira e Campomar (2007) definem que o posicionamento consiste no desenvolvimento e na transmissão (alicerçada em uma marca) de uma proposta de valor, a partir de aspectos significativos para um determinado público-alvo, os quais serão processados e comparados aos concorrentes, originando o posicionamento percebido.

Do ponto de vista de posicionamento de produto, Aaker e Shansby (1982, p.56-58) apontam seis possibilidades: atributo do produto, relação qualidade preço, uso ou aplicação, associação com usuários, classe de produto e relação com competidores.

De fato identificou-se que o posicionamento pode ser analisado e discutido como posicionamento de mercado e posicionamento psicológico (PORTER, 2004; AAKER; SHANSBY, 1982; TOLEDO; SIQUEIRA, 2001; DI MINGO, 1988; CRAVENS; PIERCY, 2007; HOOLEY; SAUNDERS; PIERCY, 2005; RIES; TROUT, 2003), do ponto de vista de marca, produto e empresa (AAKER D.; 1982-1991-2001-2002; AAKER J.; 1997; PORTER 2004; CRAVENS; PIERCY, 2007; HOOLEY;SAUNDERS; PIERCY, 2005; RIES; TROUT, 2003) além de posicionamento almejado e percebido (OLIVEIRA; CAMPOMAR, 2007).

Os fatores para o desenvolvimento de relacionamento de longo prazo com clientes podem diferir um pouco em relação a produtos tecnológicos. Determinadas variáveis que influenciam o comportamento dos consumidores sobre à intenção de se usar tecnologias específicas estão mais relacionadas aos fatores: utilidade da tecnologia; a facilidade com a qual a tecnologia pode ser usada e aspectos sociais relacionadas a tecnologia (PARASURAMAN, 2000; PARASURAMAN; COLBY, 2002, Davis (1989); Swilley (2010). E, como o posicionamento diz respeito à percepção que clientes 
potenciais têm sobre um produto, é fundamental conhecê-los em termos de interesses, atitudes e comportamentos em relação à tecnologia.

Em vista disso, Parasuraman e Colby (2002, p. 32-44), criaram um indicador denominado Technology Readiness Index (TIR). Trata-se de uma combinação de crenças em relação às inovações que, conjuntamente, determinam a predisposição para lidar com uma nova tecnologia. Os autores identificaram quatro facetas de disponibilização para tecnologia: duas são contribuintes e definidas como otimista e caráter inovador, pois aumentam a disposição da pessoa para tecnologia, e duas são inibidoras e definidas como desconforto e insegurança, pois reduzem a disposição para tecnologia, conforme descrito na Figura 1 (PARASURAMAN; COLBY, 2002).

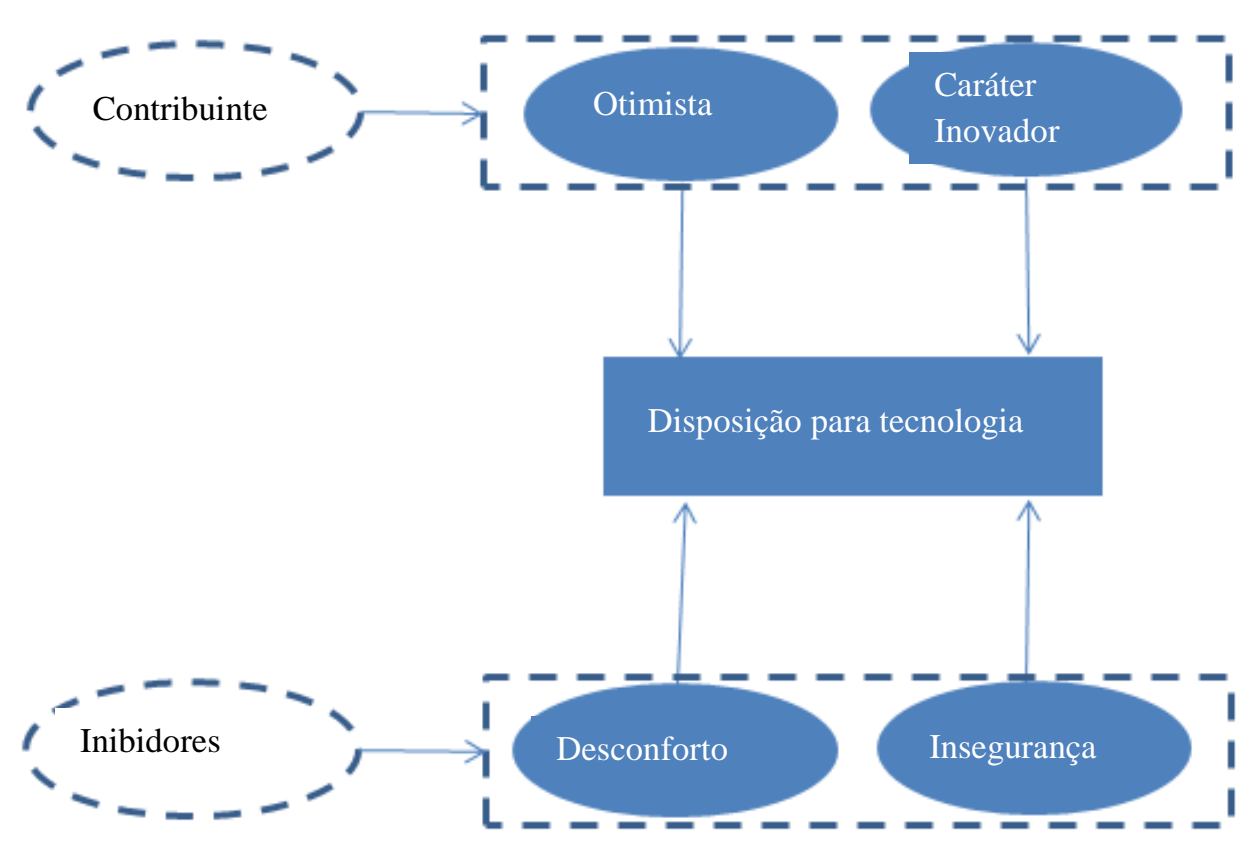

Figura 1 - Impulsionadores de disponibilização para tecnologia Fonte: Parasuraman e Colby (2002, p.37).

Os mesmo autores também identificaram cinco tipos de clientes de tecnologia que podem ser combinadas com os quatro tipos de atitude apontados anteriormente, a saber: os exploradores ou os primeiros a chegar; os pioneiros desejam os benefícios dos novos produtos, mas tem maior praticidade em relação às dificuldades e perigos; os céticos que precisam ser convencidos dos benefícios; os paranóicos que apesar de se convencer dos benefícios demonstram enorme preocupação com os riscos do novo conceito; e os retardatários que podem nunca vir a adotar a tecnologia a não ser que não tenham escolha (PARASURAMAN; COLBY, 2002). 
Souza e Luce (2005, p. 126-128) fizeram uma análise sobre a adequação do TIR aos consumidores brasileiros e concluíram haver seis perfis atitudinais, e não quatro: otimista, caráter inovador, insegurança com a informação, desconforto com o constrangimento, insegurança pela falta de contato pessoal e desconforto com o risco funcional e físico.

A seguir é apresentada a metodologia da pesquisa de campo.

\section{METODOLOGIA DA PESQUISA DE CAMPO}

Tendo por objetivo conhecer o posicionamento de novos produtos com inovação tecnológica, $\mathrm{e}$ o atual estágio de discussão em que o tema se encontra na literatura, definiu-se a pesquisa conclusiva descritiva como a mais apropriada, a qual foi realizada por meio de um estudo de caso.

Para Yin (2005) apesar de muitos cientistas acreditarem que um estudo de caso só é aplicável à fase exploratória de uma pesquisa, a visão hierárquica das estratégias de pesquisa, ou seja, exploratória, descritiva e experimentos, são questionáveis, pois sempre houve experimentos por motivos exploratórios, uma vez que o desenvolvimento de explanações causais pode ser refletido na historiografia e o estudo de caso não é apenas uma estratégia exploratória.

A pesquisa descritiva expõe características de determinado fenômeno, mas não tem compromisso de explicar o fenômeno que descreve embora sirva de base para tal explicação (VERGARA, 2009, p. 42).

O estudo de caso é uma forma distinta de metodologia empírica adequada a responder perguntas de "como" e "por que" que não exige controle sobre eventos comportamentais e focaliza acontecimentos contemporâneos (YIN, 2005).

O estudo de caso pode ser adotado para um caso único ou para casos múltiplos e possuem aplicação nas questões relacionadas a explicar, descrever, ilustrar, explorar e pode ser uma metaavaliação (YIN, 2005, p.33-35).

A figura 2, abaixo relacionada, ilustra os tipos básicos de projetos de estudos de caso: 


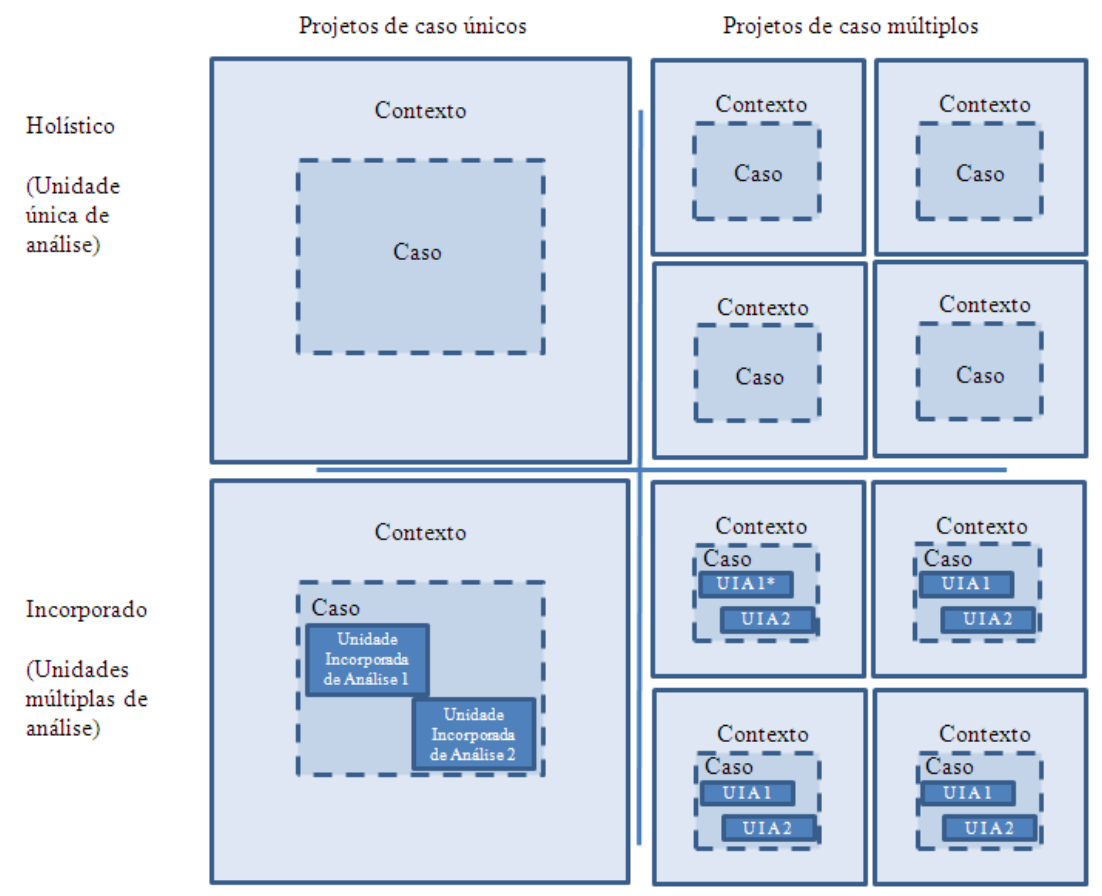

Figura 2 - Tipos básicos de projetos para estudos de caso Fonte: COSMOS Corporation. apud Yin, 2005

O estudo de caso único justifica-se neste trabalho, pois o mesmo pode ser tomado como um estudo inicial gerando oportunidade de se realizarem novos estudos baseado em múltiplos casos relacionados a posicionamento de produtos tecnológicos. A pesquisa teve como base um estudo de caso único, que "pode representar uma importante contribuição à base de conhecimento e a construção da teoria" e pode, também, ter na sua lógica de fundamentação a representação de um caso raro ou extremo (YIN, 2005, p.62). O mesmo autor aponta mais três fundamentos para a escolha de um estudo de caso único (YIN, 2005,p. 63-64):

- Caso representativo ou típico: Pode representar um projeto típico dentro de muitos projetos diferentes;

- Caso revelador: Estudo de caso previamente inacessível a investigação científica;

- Caso longitudinal: Estudar o mesmo caso em momentos temporais distintos.

A decisão de escolha de estudo de caso único teve como base os fundamentos 1 e 4 uma vez que a revisão bibliográfica revelou carência da literatura sobre o objeto em questão. Tem-se para o caso que foi estudado o contexto de produto tecnológico e como posicioná-los estrategicamente.

O caso estudado teve como objeto um produto denominado WI-FI, lançado por uma empresa de telecomunicações. 
A coleta de dados foi realizada por meio de entrevista com a gerente responsável pelo produto, pois ela possuía responsabilidade sobre o posicionamento dele. Utilizou-se um roteiro semiestruturado, elaborado com base na revisão da literatura.

A entrevista foi gravada, mediante permissão da pesquisada, e posteriormente realizou-se a sua transcrição. A análise foi descritiva e interpretativa, buscando-se verificar adequação ao padrão.

Foram utilizadas as seguintes fontes de evidência para triangulação dos dados:
a) Manual de desenvolvimento de novos produtos;
b) Entrevista com a gerente de produtos responsável pelo WI-FI;
c) Documentos gerenciais;
d) Observação em campo.

Os nomes da empresa e da entrevistada não foram autorizados a serem divulgados.

\section{APRESENTAÇÃO DOS RESULTADOS}

\subsection{Apresentação da Empresa e do Produto}

A empresa pesquisada atua no Estado de São Paulo como concessionária, e é autorizatária dos demais serviços de comunicações que oferece. Pertence a um grande grupo europeu.

A empresa está dividida em quatro áreas de negócios. Todas as áreas respondem a um diretor geral e são hierarquicamente divididas em diretores executivos e gerentes. As quatro áreas de negócios atendem, respectivamente, aos seguintes segmentos de mercado: a) residencial (massivo); b) negócios (pequenas e médias empresas); c) empresas (grandes empresas) e d) atacado (outras operadoras).

A empresa é líder de mercado nos segmentos que atua. Na Tabela 1 podem ser observados alguns dados da empresa.

Tabela 1 - Dados operacionais da empresa pesquisada

\begin{tabular}{|c|c|c|c|}
\hline Indicadores (2011) & Mundo & Brasil & \% BR/Mundo \\
\hline Receita Líquida & $€ 56,7$ bilhões & $\mathrm{R} \$ 23,1$ & $14,8 \%$ \\
\hline Número de empregados & 264,6 mil & 97,2 mil & $36,7 \%$ \\
\hline Clientes (Total de acessos) & 277,4 mil & 73,2 milhões & $26,3 \%$ \\
\hline Clientes de telefonia fixa & 41,4 milhões & 11,3 milhões & $27,2 \%$ \\
\hline Clientes de telefonia móvel & 214,8 milhões & 57,7 milhões & $26,8 \%$ \\
\hline Clientes de dados e internet $*$ & 18,3 milhões & 3,7 milhões & $20,2 \%$ \\
\hline Clientes de TV paga & 2,7 milhões & 466 mil & $17 \%$ \\
\hline Clientes de Atacado & 4,4 milhões & 33,8 mil & $0,7 \%$ \\
\hline
\end{tabular}

Fonte: Documento fornecido pela empresa pesquisada. 
O caso tem por objeto o lançamento de um produto denominado WI-FI. Trata-se de uma solução de acesso sem fio à Internet banda larga. O cliente porta um dispositivo de acesso sem fio que pode ser um notebook, ou um smarthphone, identifica se há existência da rede WI-FI no estabelecimento que se encontra, e escolhe o meio de acesso através de um portal.

É uma solução de mobilidade e há rede de acesso em diversos estabelecimentos comerciais e lugares públicos. A empresa pesquisada é pioneira na implantação de redes sem fio com tecnologia WI-FI, contando atualmente com mais de 150 hot-spots instalados em cafés, universidades, shoppings centers, restaurantes, hotéis, centro de convenções e aeroportos. A rede de WI-FI da empresa está presente no Brasil e em diversos países da América Latina e Europa. Na Figura 3 é apresentada a arquitetura do produto.

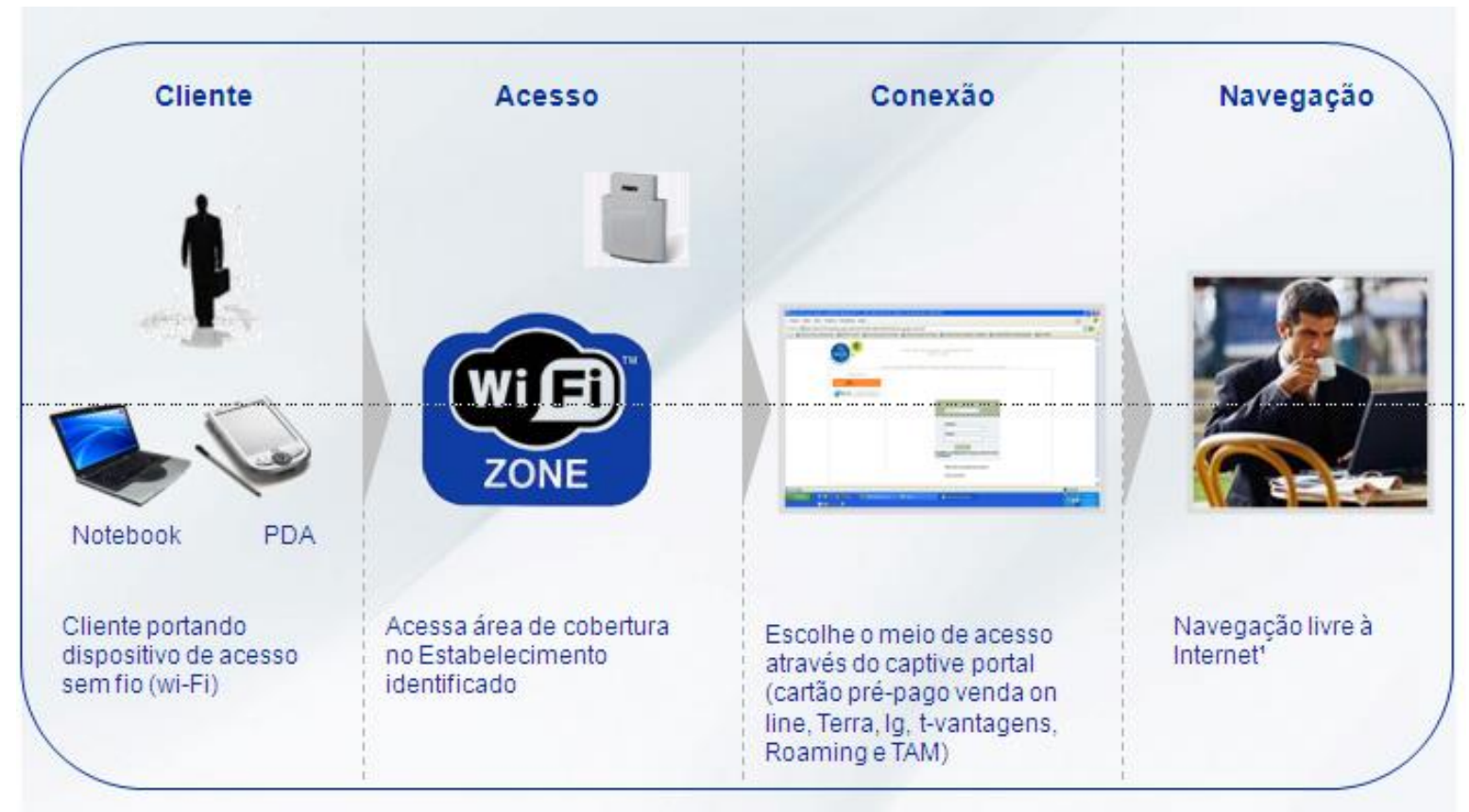

Figura 3 - Arquitetura do produto WI-FI

Fonte: Documento disponibilizado pela empresa pesquisada.

\subsection{Descrição do Caso}

Questionada sobre a estratégia geral de posicionamento definido para o produto WI-FI, a entrevistada relatou que, por ele ser voltado a diversos públicos, tem por foco o acesso à Internet em qualquer lugar. Menciona, ainda, que para certos produtos define-se "uma estratégia de atuação diferenciada”.

No processo de desenvolvimento de produto adotado pela empresa, as definições sobre posicionamento são tomadas no início do desenvolvimento. Quando se parte para as análises financeiras de viabilidade, as definições de posicionamento já foram decididas. Segundo a executiva 
“já está concebido; quando vai para as pesquisas, já devo saber qual o posicionamento que pretendo ocupar; na hora de fazer o business plan isso já deve estar definido”.

A executiva entende que o posicionamento do produto é parte fundamental das definições iniciais da estratégia e que as demais etapas são operacionais. De acordo com ela, "No início do processo devemos ter as definições de posicionamento todo processo de inteligência; dai para frente é linha de produção”.

Pôde-se notar, inclusive, a importância que a empresa dá à questão, pois no Manual de Desenvolvimento de Produtos e Serviços da empresa, consta preocupação específica com o posicionamento de novos produtos.

A estratégia de posicionamento estava relacionada à busca de fidelização, fazendo com que seus usuários de banda larga percebessem mais valor na solução. $\mathrm{O}$ posicionamento tinha como premissa ser percebido pelo cliente como um produto de alta qualidade com mobilidade, através da tecnologia WI-FI. Devido à inovação incorporada e ser a primeira empresa a oferecer o serviço de maneira massificada e de forma bastante abrangente em termos geográficos, não houve preocupações específicas com a concorrência.

Pôde-se notar que o posicionamento foi baseado em múltiplos fatores, conforme possibilidades apontadas por Aaker e Shansby (1982): qualidade-preço, uso-aplicação e usuário do produto.

A executiva citou que o posicionamento dos novos produtos deve ser compatível com o posicionamento de outros produtos e com o posicionamento institucional, conforme apontado por Oliveira e Campomar (2007).

Quanto à percepção do posicionamento por parte do mercado, a entrevistada mencionou haver dissonância em relação ao posicionamento pretendido para o produto. Segundo ela,

Queríamos fazer uma diferenciação, convidar o usuário a usar mais... trabalhamos o preço, tentamos demonstrar ao cliente que era muito fácil e não era custoso ele fazer a utilização do WI-FI...o objetivo era fazer com que ele percebesse esse valor no produto...e no fim ele não capturou nada.

Quando a dissonância foi diagnosticada, a estratégia foi alterada, buscando-se então não mais novas vendas, mas sim a fidelização dos clientes. De acordo com a entrevistada foram avaliadas as dimensões relacionadas ao mercado consumidor, concorrência e empresa acerca do produto WI-FI no que tange as decisões de posicionamento; mas não foi trabalhada a dimensão "outros públicos", conforme preconizado no modelo de Oliveira e Campomar (2007).

Observou-se na literatura (PARASURAMAN; COLBY, 2002), que existem diversos grupos e segmentos de clientes quando se trata de produtos tecnológicos e que esses clientes são divergentes 
quando analisadas as diversas fases de familiaridade com tecnologia, o que remete a refletir sobre a forma de se posicionar novos produtos com inovação tecnológica.

Para a entrevistada, no que diz respeito ao posicionamento de produtos tecnológicos, o mais importante não é saber como abordar cada um dos grupos, mas sim fazer um convite a toda sociedade a conhecer o mundo da tecnologia. A empresa busca colocar todos os seus potenciais clientes inseridos no contexto tecnológico, mas não se preocupa em entender os diversos grupos de clientes, as suas necessidades, os seus medos em usar produtos tecnológicos. Por outro lado, propõe debates sobre o uso da tecnologia para melhorar a vida das pessoas.

$\mathrm{Na}$ entrevista a executiva relata que não há uma preocupação da empresa em ter um diálogo diferente com um público que não é tecnológico, o que se pratica é um diálogo simples para que todos possam saber da existência das possibilidades tecnológicas oferecidas ao mercado.

A preocupação da empresa é colocar todo mundo inserido no contexto da tecnologia, ela não vai deixar de falar com um ou com outro porque eles não são tecnológicos; ela vai convidá-los ao mundo da tecnologia contando o que é, como é, trazendo um pouco desse conhecimento e da facilidade em entrar neste mundo e o quão gostoso é estar dentro dele.

A executiva entende que a estratégia deveria ser diferente para os diversos segmentos, e se caso a empresa não tratasse de forma abrangente as abordagens sobre uso de tecnologia, haveria maior necessidade de segmentação e estratégias de posicionamento aos diversos tipos de consumidores. Porém, para alguns casos pontuais em se tratando de um produto específico, as estratégias são tratadas de forma diferenciada "se for um produto exclusivo teremos uma estratégia de atuação diferenciada o que não é o caso do WI- FI que era um convite a qualquer um para entrar no mundo tecnológico”.

A executiva relatou que a sua percepção revela que um produto tecnológico não tem necessariamente um ciclo de vida menor que outros demais produtos. Na visão dela, o que acontece com os produtos tecnológicos são constantes necessidades de adequações tecnológicas, fazendo-os sobreviver às mudanças e exigências do mercado. Para isso, o posicionamento não pode ser pautado em algo que tende a envelhecer logo.

Nas palavras dela,

Os produtos tecnológicos necessitam de mais flexibilidade em função das novas tecnologias que surgem e em função das oportunidades de complementação, ou até eventualmente a possibilidade em se fazer uma mudança tecnológica e atualizações.

A entrevistada relatou que o produto $W I-F I$ sofreu diversas adequações na sua tecnologia, mas que o posicionamento permaneceu o mesmo. 
Em geral os seus produtos são todos tecnológicos, pois como já discutiu-se antes, trata-se de uma empresa de telecomunicações, dentro de um setor em transformação, apontando para empresas de soluções digitais e não mais de telecomunicações somente. Nota-se dentro do portfólio de produtos de diversas empresas que competem neste setor, uma mudança na oferta, relacionado, por exemplo, à inserção de TV digital, mobilidade, banda larga, soluções para smart phone, transmissão de dados para diversos tipos de transações, composição de redes de fibra óptica e backbones cada vez maiores do ponto de vista da capacidade de transmissão de dados, voz e imagem.

$\mathrm{Na}$ entrevista a executiva relata que não há uma preocupação da empresa em ter um diálogo diferente com um público que não é tecnológico, o que se pratica é um diálogo simples para que todos possam saber da existência das possibilidades tecnológicas oferecidas ao mercado.

\begin{abstract}
A preocupação da empresa é colocar todo mundo inserido no contexto da tecnologia, ela não vai deixar de falar com um ou com outro porque eles não são tecnológicos; ela vai convidá-los ao mundo da tecnologia contando o que é, como é, trazendo um pouco desse conhecimento e da facilidade em entrar neste mundo e o quão gostoso é estar dentro dele.
\end{abstract}

\title{
5. CONCLUSÕES
}

Este trabalho teve por objetivo conhecer o posicionamento de novos produtos com inovação tecnológica. Além da revisão da literatura foi realizada uma pesquisa conclusiva descritiva, pelo método do estudo de caso.

Pôde-se observar que a empresa considera as possibilidades e a prática do posicionamento conforme identificados na literatura, com algumas importantes diferenças. Uma delas é a consideração limitada da concorrência, talvez devido ao fato de que a inovação, por sua própria natureza, pressupõe diferenciais importantes em relação ao que existe no mercado. A outra diz respeito à preocupação em se estabelecer um posicionamento focado em variáveis que sejam menos perecíveis e, especialmente, que não sejam suscetíveis a abalos decorrentes de modificações e evoluções, já que estas são práticas constantes e necessárias em produtos dessa natureza.

O posicionamento focado em mobilidade e qualidade buscou exatamente atenuar a questão da atualização, que inclusive foi realizada pela empresa. Assim, conforme mencionado pela própria executiva entrevistada, o posicionamento é o mesmo ao longo do tempo.

Pode-se perceber que a empresa trata consumidores com disponibilização para tecnologia de forma diferenciada dos consumidores que não tem disposição para tecnologia.

Revista de Administração e Inovação, São Paulo, v.12, n.1, p.52-69, jan./mar. 2015. 
No caso apresentado, a empresa se preocupa em fazer um convite a toda à sociedade a usufruir da tecnologia por consequência de diversos produtos ofertados no seu portfólio, mas não se preocupa em entender as características dos consumidores que apresentam algum tipo de desconforto ou inibição com tecnologia (PARASURAM; COLBY, 2002).

Quando uma análise relacionada aos cinco tipos de clientes com disponibilização para uso de tecnologia, identificados na teoria como exploradores, pioneiros, céticos, paranóicos e retardatários, também é perceptível a postura da empresa quanto a sua preocupação com os clientes exploradores e pioneiros e ausência de estratégias para conquista de clientes céticos, paranóicos e retardatários. Como descrito na entrevista, a empresa faz um convite à sociedade para usar tecnologia o que parece de fácil aceitação aos usuários com perfil explorador e pioneiro (PARASURAM; COLBY, 2002).

Os fatores críticos de sucesso apresentados na revisão da literatura parecem fazer sentido quando verificamos para este caso as seguintes evidências (PARASURAMAN; COLBY, 2002):

- A empresa realmente trata o processo de adoção de tecnologia de forma diferenciada, ofertando ao cliente vivenciar a experiência

- Os primeiros adotantes tem papel fundamental na adoção desses produtos tecnológicos.

- Podemos notar a preocupação da empresa com a qualidade dos serviços ofertados, a sua necessidade de blindagem e fidelização de clientes atuais ofertando valor adicional ao principal produto de banda larga através de mobilidade.

- A regra de "o vencedor fíca com tudo" tratando-se de produtos tecnológicos a posição dominante raramente é ameaçada, tal fato, torna-se evidente ao notarmos minimizações com a preocupação das ameaças da concorrência em relação ao produto pesquisado.

Pode-se notar na pesquisa e especificamente para o caso apresentado que além das dimensões verificadas na literatura relacionadas a critérios de posicionamento (AAKER, 1982), a empresa adotou para o produto pesquisado uma estratégia de fidelização através do posicionamento almejado de mobilidade e qualidade em relação ao seu principal produto ADSL, ou seja, a entrevistada identificou que poderia haver uma outra variável que considera o posicionamento voltado a fidelização e blindagem dos clientes atuais. Este pode ser um indício que a fidelização para produtos tecnológicos faça sentido quando existe maior percepção de valor ao produto, ou buscar o estágio de produto potencial ofertando produtos com benefícios adicionais ainda não oferecidos pela concorrência ( HOOLEY; SAUNDERS; PIERCY, 2005).

Revista de Administração e Inovação, São Paulo, v.12, n.1, p.52-69, jan./mar. 2015. 
Também para pesquisas futuras, sugerimos a aplicação do estudo de caso aqui proposto em outras empresas do setor de telefonia fixa e novas pesquisas sobre como posicionar produtos tecnológicos em outros setores da indústria com a recomendação de evidenciar se as características dos clientes que adotam tecnologia são as mesmas nos diversos setores da indústria.

Em vista da escassez de trabalhos que abordam especificamente o marketing aplicado a produtos com inovação tecnológica, sugere-se que outros estudos que o abordem sejam realizados, focando, além do posicionamento (como foi o caso deste), as diversas atividades e ferramentas do processo de marketing.

\section{REFERÊNCIAS}

AAKER, David A. Administração estratégica de mercado. Porto Alegre: Bookman, 2001.

AAKER, David A.; SHANSBY J. G. Positioning your product. Business Horizons, Greenwich, v. 25, n. 3, p. 56-62, May/June 1982.

ARRUDA, M. R.; VERMULM, S.; HOLLANDA, S. Inovação tecnológica no Brasil: A indústria em busca da competitividade global. São Paulo: ANPEI, 2006.

BAINSFAIR, P. Create your own context and find a place in the public mind. Compaign, 16 Nov. 1990.

CRAVENS, David W.; PIERCY, Nigel F. Marketing estratégico. 8 ed. São Paulo: McGraw-Hill, 2007.

DAVIS, F.D. Perceived usefulness, perceived ease of use, and user acceptance of information technology. MIS Quaterly, v. 13, n.3, p. 319-40, 1989

DAY, George S. A empresa orientada para o mercado: compreender, atrair e manter clientes valiosos. Porto Alegre: Bookman, 2001.

DI MINGO, E. The fine art of positioning. The Journal of Business Strategy, Boston, v. 9, n. 2, p.3438, Mar./Apr. 1988.

DOGSON, M.; GANN, D.; SALTER, A. The Management of Technological Innovation: Strategy and Practice. New York: Oxford Univerty Press, 2008.

DRUCKER, P. Inovação e espírito empreendedor: Prática e príncipios. São Paulo: Pioneira Thomson Learning, 2003.

ESTHER, S. Technology Rejection: The case of wallet phone. Journal of Consumer Marketing, v. 27/4, p.304-12, 2010.

Revista de Administração e Inovação, São Paulo, v.12, n.1, p.52-69, jan./mar. 2015. 
FREEMAN, C.; SOETE, L. The Economics of Industrial Innovation. London: Pinter, 2007.

GHEMAWAT P. A estratégia e o cenário de negócios. Porto Alegre: Bookman, 2007.

HOOLEY, Graham J.; SAUNDERS, John A.; PIERCY, Nigel F. Estratégia de Marketing e Posicionamento Competitivo. São Paulo: Pearson Prentice Hall, 2005.

KALAFATIS, Stravos P.; TSOGAS, Markos H.; BLANKSON, C.; Positioning strategies in business markets. The Journal of Business \& Industrial Marketing, v. 15, p. 416-429, 2000.

KIM, W. Chan; MAUBORGNE, Renée. A Estratégia do Oceano Azul: Como criar novos mercados e tornar a concorrência irrelevante. Rio de Janeiro: Campus, 2005

LEVITT, Theodore. Marketing myopia. Harvard Business Review, v. 38, p. 45-56, jul/ago, 1960.

MAGGARD, J. P. Positioning revisited. Journal of Marketing, New York, v. 40, n. 1, p. 63-66, Jan. 1976.

MATTAR, F.N. et al. Gestão de produtos, serviços, marcas e mercados: estratégias e ações para manter-se top of market. São Paulo: Atlas, 2009.

MÜHLBACHER, H.; DREHER, A.; GABRIELRITTER, A. MIPS - Managing industrial positioning strategies. Industrial Marketing Manegement, New York, v. 23, n. 4, p. 287-297, Oct. 1994.

OGILVY, D. Ogilvy on Advertising. London: Ramdon House Inc., 1985.

OLIVEIRA, Braulio; CAMPOMAR, Marcos C. Revisitando o posicionamento em marketing. Revista de Gestão da USP. São Paulo, v. 14, n. 1, p. 41-52, jan./mar. 2007.

OLIVEIRA, Braulio; MOTTA, Sérgio L. S. Segmentação de mercado e posicionamento da oferta. In: OLIVEIRA, Braulio. Gestão de Marketing. São Paulo: Pearson, 2011.

ORGANISATION FOR ECONOMIC CO-OPERATION AND DEVELOPMENT- OECD. Manual de Oslo: Diretrizes para coleta e interpretação de dados sobre inovação. 3. ed. Rio de Janeiro: Finep, 2005.

PARASURAMAN, A. Technology Readiness Index (TRI): a multiple-item scale to measure readiness to embrace new technologies. Journal of Service Research, v. 2, n. 4, p. 307-320, 2000.

PARASURAMAN, A.; COLBY, C. L. Marketing para produtos inovadores: como e por que seus clientes adotam tecnologia. Porto Alegre: Bookman, 2002.

PORTER, Michael E. Estratégia competitiva: técnicas para a análise de indústrias e da concorrência. 16 ed. Rio de Janeiro: Campus, 2004.

PRAHALAD C.K.; HAMEL G. Competindo pelo futuro: estratégias inovadoras para obter o controle do seu setor e criar os mercados de amanhã. Rio de Janeiro: Campus, 2005.

RIES, Al; TROUT, Jack. Marketing de Guerra. 1 Ed. São Paulo: M.Books, 2006.

Revista de Administração e Inovação, São Paulo, v.12, n.1, p.52-69, jan./mar. 2015. 
Posicionamento: a batalha pela sua mente. São Paulo: Pioneira, 2003.

SCHUMPETER, J.A. Capitalism, Socialism and Democracy. London and New York: Routledge, 2003.

SHUGAN, S.M. The impact of advancing technology on marketing and academic and academic research. Marketing Science, v. 23, p. 469-75, 2004.

SOUZA, R. V.; LUCE, F. B. Avaliação da Aplicabilidade do Technology Readiness Index (TRI) para a Adoção de Produtos e Serviços Baseados em Tecnologia. Revista de Administração Contemporânea, v. 9, n. 3, pp. 121-141, Jul./Set. 2005.

STONEMAN, Paul; DIEDEREN, Paul. Technology diffusion and public policy. The Economic Journal, v.104, p.918-930, Jul. 1994.

TOLEDO, G. L.; HEMZO, M. A. O processo de posicionamento e o marketing estratégico. In: ENCONTRO ANUAL DA ASSOCIAÇÃO NACIONAL DE PROGRAMAS DE PÓS GRADUAÇÃO EM ADMINISTRAÇÃO, 15, 1991, Belo Horizonte. Anais... Belo Horizonte: ANPAD, 1991.

TOLEDO, G. L.; SIQUEIRA, A. C. B. Bases de segmentação de mercados no marketing industrial: um estudo de caso na indústria de elevadores. Revista de Administração, São Paulo, v. 36, n. 4, p. 52-63, outubro/dezembro 2001.

TORNATZKI, L. G.; KLEIN, K. Innovation characteristics and innovation implementation: a metaanalysis of findings. IEEE Transactions Engineering Management, v. 29, n. 1, p. 28-45, 1982.

VERGARA, S.C. Projetos e relatórios de pesquisa em administração. São Paulo: Atlas, 2009.

WIND, Y. J. Product policy: concepts, methods and strategy. Massachusetts: Addison-Wesley Publishing Company, 1982.

YIN, Robert K. Estudo de caso: planejamento e métodos. Porto Alegre: Bookman, 2005. 


\title{
STRATEGIC POSITIONING OF PRODUCTS WITH TECHNOLOGICAL INNOVATION: THE WI-FI PRODUCT CASE
}

\begin{abstract}
Possible differences in marketing applied to products with technological innovation require specific studies on the topic. But for now, few studies have been developed with this focus. This study sought to better understand the process of positioning a product with technological innovation. Besides reviewing the literature, we conducted a case study. The results show small differences from the traditional model of positioning, in particular the need to define a positioning perish, since technological products, by their very nature, require constant updates.
\end{abstract}

Keywords: Positioning; Technological innovation; New products.

Data do recebimento do artigo: 22/01/2014

Data do aceite de publicação: 08/01/2015

Revista de Administração e Inovação, São Paulo, v.12, n.1, p.52-69, jan./mar. 2015. 